\title{
Assessment, investigation, and management of acute monoarthritis
}

\author{
Simon H Till, M L Snaith
}

The theme of this series is the management of "every day" clinident and emergency departments. dent and emergency departments.
Part of the aim of the series is to Part of the aim of the series is to ing medical education (CME) ing medical education (CME)/ continuing professional development (CPD) and authors have
been asked to provide three quesbeen asked to provide three ques-
tions and key references. If you tions and key references. If you
would like to participate please send your answers to $\mathrm{Mr}$ Fim Wardrope, Associate Editor, Accident and Emergency Department, Northern General Hospital Herries Road, Sheffield S5 7AU and he will send an answer sheet. Those readers submitting answers will be $C P D$ logbook as one hour of external CME/CPD.

Leicester Royal Infirmary and Nottingham City Hospital

S H Till

Institute for Bone and Joint Medicine, Sheffield University Medical School M L Snaith

Correspondence to: Dr Simon H Till, Consultant in Rheumatology and Sport and Exercise Medicine, Royal Hallamshire Hospital, Glossop Road, Sheffield S10 2JF.

Accepted 5 May 1999

\section{Abstract}

Trauma is the commonest cause of acute monoarticular joint pain and swelling in patients attending an accident and emergency (A\&E) department. However, in a significant minority of patients there will be no history of trauma and consequently a different approach to assessment and investigation is required.

Our aim is to offer an outline of how to assess, investigate, and manage a patient with monoarthritis. Despite advances in antibiotic treatment diagnostic delay partly explains why septic arthritis is still associated with considerable morbidity and mortality. It is therefore imperative that joint infection is considered above all other diagnoses. Arthrocentesis is a relatively safe procedure and doctors in A\&E medicine are encouraged to develop the skills required to aspirate large joints.

In the same way that the A\&E department is often portrayed as the shop window of a hospital, the joint can reflect a wide variety of internal diseases. Connective tissue disease, inflammatory bowel disease, sarcoidosis, and vasculitis can all present with a monoarthritis. A nonspecific reactive monoarthritis may be a feature of a wide variety of common and uncommon infections including, brucellosis, Lyme disease, and leptospirosis. Drugs are also associated with acute arthritis either through their metabolic consequences or as idiosyncratic drug reactions.

The ability for the joint to reflect multisystem disease necessitates close liaison with specialists from other fields. A multidisciplinary approach to the management of these patients is strongly encouraged as some will have unusual diseases that require specialist advice.

It is not difficult to appreciate how the patient with monoarthritis can present the clinician with a fascinating diagnostic and therapeutic challenge, which we hope this article will help to unravel. ( $F$ Accid Emerg Med 1999;16:355-361)

Keywords: monoarthritis; infection related arthritis; rheumatology
Trauma is the commonest cause of acute joint pain and swelling in patients attending an accident and emergency (A\&E) department. However, in $5 \%-10 \%$ of patients there will be no history of injury. The spectrum of diagnosis is so different in non-traumatic joint pain that a significantly different approach is required in the assessment and investigation of such patients.

Acute pain and swelling in a joint always requires urgent assessment. Septic arthritis carries a risk of mortality and prolonged morbidity ${ }^{1}$ and therefore needs to be excluded before other causes of a monoarthritis are considered (table 1). The early assessment of a non-septic inflamed joint also provides an opportunity to confirm a diagnosis which may be lost once the inflammatory episode subsides.

Assessing the patient with monoarthritis HISTORY

Differentiation between trauma and non-trauma This is the first essential step in the assessment. Do not assume that all patients with limb pain must have an injury. Some patients may try to rationalise their symptoms (and their attendance at an $A \& E$ department) by giving a vague history of trivial injury. The absence of a clear mechanism of injury should alert the doctor that this is not a simple case of a minor trauma. These patients require a more rigorous history if important diagnoses are not to be missed.

History of the illness

Obtain a clear history of the onset of the illness, its progress, and all the patient's symp-

Table 1 Differential diagnosis of acute monoarthritis

\begin{tabular}{ll}
\hline Disease & Cause \\
\hline Infection & $\begin{array}{l}\text { Bacterial } \\
\text { Gonococcal } \\
\text { Non-gonococcal } \\
\text { Tuberculous }\end{array}$ \\
& $\begin{array}{l}\text { Fungal } \\
\text { Viral } \\
\text { Monosodium urate } \\
\text { Calcium pyrophosphate dihydrate } \\
\text { Crystal induced arthritis } \\
\text { Oxdroxyapatite } \\
\text { Oipid } \\
\text { Reactive arthritis }\end{array}$ \\
$\begin{array}{l}\text { Bacterial } \\
\text { Viral }\end{array}$ \\
Trauma & \\
Malignancy & \\
Systemic disease & \\
\hline
\end{tabular}


toms. Severe pain, especially at night, raises the question of more sinister pathology such as malignancy and infection and should put the A\&E clinician on guard. A more extensive history is needed, including eliciting systemic symptoms such as fever, weight loss, or general malaise. Reactive or septic arthritis may be suggested by a history of any recent nonarticular infections, for example bowel, upper respiratory tract and genitourinary, ${ }^{2}$ sexual risk factors, travel abroad, and intravenous drug use. Conjunctivitis, urethritis, and diarrhoea might also point to a reactive arthritis. A history of previous episodes or appropriate risk factors (commonly alcohol or diuretic treatment) may provide some support for a crystalline arthritis and a history of back pain, psoriasis, or inflammatory bowel disease and relevant family history may point to a seronegative spondyloarthritis.

A number of multisystem diseases can present with a monoarthritis. Bloody diarrhoea, weight loss, and symptoms of malabsorption may indicate inflammatory bowel disease. It should be appreciated that occasionally arthritis may be the only clinical indicator of occult inflammatory bowel disease. A history of Raynaud's disease, mouth ulcers, alopecia, or skin rashes (particularly in a photosensitive distribution) could indicate the presence of a number of connective tissue disease, for example systemic lupus erythematosis. The early stages of sarcoidosis can present with acute arthritis associated with erythema nodosum, although infections (usually streptococcal) and drugs (sulphonamides) remain the commonest causes. Vascultic syndromes, for example Wegener's granulomatosis, polyarteritis nodosa, and giant cell arthritis can also present with monoarthritis. Non-specific symptoms such as weight loss and lethargy in conjunction with specific symptoms like skin ulceration, purpuric skin rashes, myalgia, and recent onset upper and lower airway symptoms should alert the clinician to the possibility of vasculitis and the importance of looking for occult involvement of major organs, particularly the kidneys (urine dipstick for blood and protein is mandatory). Although all of these conditions should be included in any differential diagnosis, most of them are uncommon causes of monoarthritis and confirmation of the diagnosis will require referral to an appropriate specialist for further investigation.

\section{PHYSICAL EXAMINATION \\ General}

Grave errors may be made if the whole patient is not examined. Does the patient look ill? Is the patient pyrexial or tachycardic? Are there any other signs, for example jaundice, anaemia, or tachypnoea? Ocular inflammation is associated with Reiter's syndrome, connective tissue disease, and vasculitis. Mouth ulcers are seen in systemic lupus erythematosus and Behçet's disease. Small patches of psoriasis in the scalp, between the buttocks, or behind the ears may have escaped the patient's notice. Erythema nodosum is associated with an acute arthritis and may suggest recent infection, a drug reac-

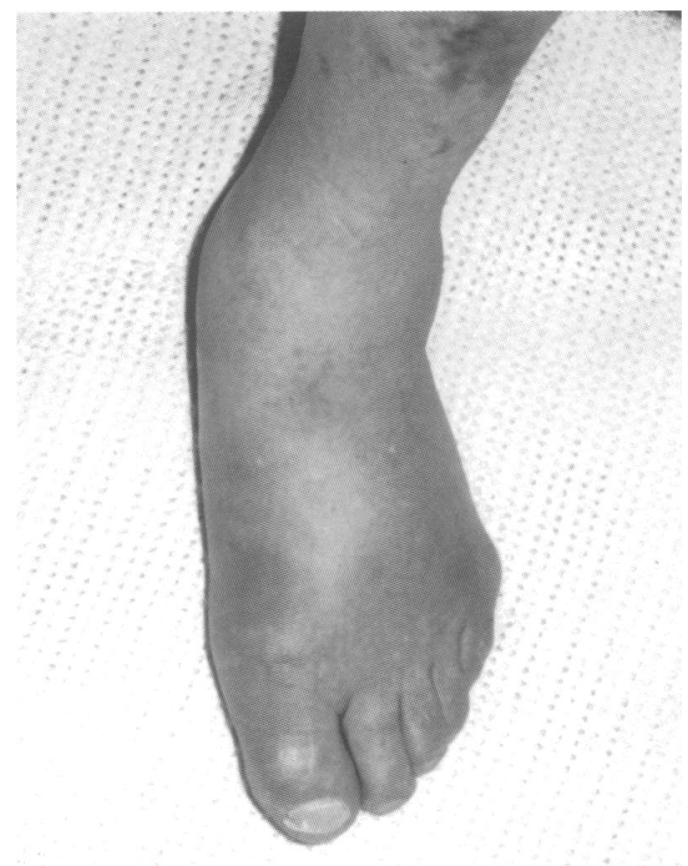

Figure 1 Typical appearance of gout. Classical popagra.

tion, sarcoidosis, or inflammatory bowel disease. The hands and feet may reveal splinter haemorrhages, nail fold infarcts, and purpuric lesions secondary to vasculitis or bacterial endocarditis. ${ }^{3}$

\section{Foint examination}

The typical features of monoarticular arthritis are listed in table 2 . Unfortunately pain, swelling, and erythema around a joint are not always indicative of arthritis, which by definition involves the articular space. Cellulitis overlying a joint, inflammation in periarticular structures such as tendons or bursae, and venous thrombosis can all mimic arthritis and should be considered. The presence or absence of the cardinal signs of inflammation will not always allow the differentiation of septic and nonseptic arthritis, although the site of the joint involved may offer a clue, for example the hot red swollen first metatarsophalangeal joint classically associated with gout (fig 1).

\section{INVESTIGATIONS}

Arthrocentesis is a relatively safe, simple procedure ${ }^{4}$ that provides crucial information which will allow an accurate diagnosis in the majority of patients. ${ }^{5}$ Most rheumatologists advocate a non-touch technique which involves identifying the joint line, marking the overlying skin with the blunt end of the needle sheath, cleaning the skin with an appropriate antiseptic fluid, and then inserting the needle without touching the puncture site. Although it is

Table 2 Signs and symptoms of monoarthritis

Joint line tenderness

Restricted, painful joint movement Erythema

Local increase in skin temperature

Joint and soft tissue swelling

Loss of joint function

Periarticular muscle wasting 
fulfilling to aspirate a large volume of synovial fluid, there can often be enough fluid within the needle of an apparently dry tap to confirm the presence of crystals or infection. In such cases it is usually worth while sending the needle in a sterile sealed container, with an appropriately completed request form, to the laboratory.

Synovial fluid should be sent in a sterile container for microscopy, culture, and sensitivities. A second fresh sample should be sent for cytology. Synovial fluid will clot and consequently a bottle containing anticoagulant should be used. A paediatric lithium heparin (orange top) bottle is ideal as it avoids the problems with crystal analysis that will be encountered if oxalate, fluoride, or EDTA anticoagulants are used.

Blood should be obtained for full blood count, acute phase reactants (erythrocyte sedimentation rate, plasma viscosity, or $\mathrm{C}$ reactive protein), and biochemical analysis for urate, renal, and liver function tests. Blood cultures may be positive in up to $50 \%$ of cases of septic arthritis and can prove invaluable in confirming antibiotic sensitivities.

Plain radiographs are not usually helpful but nevertheless should be arranged as fractures, osteomyelitis, pre-existing joint disease, and tumours may be identified. Ultrasound is a useful way of demonstrating an effusion. If there is a high index of suspicion about the likelihood of sepsis then it can prove invaluable in obtaining synovial fluid if blind arthrocentesis has been unsuccessful. Magnetic resonance imaging does not have a role in the routine assessment of a monoarthritis but has been shown to be superior to other modalities in the assessment of sepsis in deep joints, for example sacroiliac joints. ${ }^{6}$

The culture and Gram's stain of urine as well as swabs taken from the throat, skin lesions, cervix, and urethra are important in suspected septic or reactive arthritis. Serum should also be taken for appropriate serological testing, for example antistreptolysin titre.

The management of the acute hot joint has been the subject of recent guidelines issued by the Royal College of Physicians. ${ }^{7}$ Monoarthritis represents infection until proved otherwise and therefore synovial fluid analysis is considered mandatory in the majority of cases. This view has been tested in a recent study from Newcastle, which demonstrated that there was a tendency for the outcome to be worse where the guidelines were followed in full. ${ }^{8} \mathrm{~A}$ more pragmatic approach may therefore be appropriate with arthrocentesis essential in those patients where septic arthritis is considered a probability on the basis of clinical findings and test results. If another diagnosis seems more likely on the basis of history and examination and the patient is systemically well with no pyrexia then a trial of non-steroidal antiinflammatory agents may be advised (providing there are no contraindications). This course should be short and the patient reviewed urgently (within 24-48 hours) by the general practitioner, $A \& E$ physician, or rheumatologist. Many rheumatology departments already run fast track clinics for patients with acute monoarthritis or polyarthritis.

\section{Differential diagnosis of acute monoarthritis}

INFECTION

Septic arthritis can broadly be divided into gonococcal and non-gonococcal infection.

\section{Gonococcal septic arthritis}

Gonococcal arthritis mainly affects young adults with a threefold higher incidence in women. ${ }^{9}$ A migratory tendinitis or arthritis often precedes the monoarthritis, which usually affects large joints, for example knee, wrist, and ankle. ${ }^{10}$ This should not be confused with the reactive arthritis that often follows gonorrhoea. Culture results can be helpful in this respect as strains of Neisseria gonorrhoeae that disseminate tend to have a unique membrane protein and a transparent appearance on culture. ${ }^{11}$

Isolation of the organism from the genital tract is successful in $50 \%-80 \%$ of cases, although it is essential to search for evidence of pharyngeal and rectal infection. Blood cultures may be positive during the early bacteraemic stage when the painless skin lesions are seen. Unfortunately this usually precedes the development of monoarthritis, when synovial fluid samples yield positive isolates in only $30 \%$ of patients. In suspected, but culture negative cases, the recent development of the polymerase chain reaction may provide a useful diagnostic test. ${ }^{12} 13$ Suspected cases must be referred to a genitourinary medicine department to exclude coexisting infections and arrange contact tracing.

Fortunately gonococcal septic arthritis usually responds rapidly to intravenous antibiotics and therefore tends to be less destructive than non-gonococcal arthritis. Ciprofloxacin is now the mainstay of treatment.

\section{Non-gonococcal septic arthritis}

Non-gonococcal septic arthritis tends to be a disease of young children, the elderly, and people who either immunosuppressed or have damaged or prosthetic joints. Over $80 \%$ of cases of infections are monoarticular, although polyarticular involvement is increasingly recognised in the presence of predisposing conditions such as rheumatoid arthritis. ${ }^{14}$ Large joints are most frequently affected, although any joint can become infected, for example seeding from an infected needle to the sternoclavicular joint in intravenous drug abusers. The agents responsible for non-gonococcal infection are illustrated in table 3 . The majority are Gram positive aerobes. Gram negative and anaerobic infections are increasingly associated with parenteral drug use, the immunocompromised, and with trauma. ${ }^{15}$

Early intravenous antibiotic treatment reduces joint damage. Patients should therefore be admitted for antibiotics which, pending blood and synovial culture results, reflect the likely causative organisms. ${ }^{16}$ Flucloxacillin $2 \mathrm{~g}$ four times a day will suffice for most young $(<50)$ patients and cefotaxime $2 \mathrm{~g}$ three times a 
Table 3 Causes of non-gonococcal septic arthritis

\begin{tabular}{ll}
\hline Staphylococcus aureus & $40 \%-50 \%$ \\
Staphylococcus epidermis & $10 \%-15 \%$ \\
Gram negative bacteria & $15 \%-20 \%$ \\
Non-group A, B haemolytic streptococci & $15 \%$ \\
Anaerobic bacteria & $<5 \%$ \\
Streptococcus pneumoniae & $<5 \%$ \\
Haemophilus influenzae & $<5 \%$ \\
\hline
\end{tabular}

day for older patients. Any decision must, however, reflect the clinical situation, for example history of dog bite, intravenous drug abuse, immunocompromised patient. Where there are such "special circumstances" expert microbiological advice should be requested. Effective drainage of bacteria and inflammatory products may improve the response to treatment. Arthroscopy and formal joint irrigation should be considered when the pretreatment history is long or in cases where symptoms are getting worse despite antibiotics.

The recent increase in the incidence of pulmonary tuberculosis has not, so far, been parallelled by an increase in tuberculous arthritis. Nevertheless mycobacterial infections can present with an acute arthritis. ${ }^{17}$ Axial and large weight bearing joints are most commonly affected. ${ }^{18}$ Predisposing factors, for example being immunocompromised, debilitation, and keeping tropical fish should always be sought. Chemotherapy with isoniazid and either rifampicin or pyrazinomide is usually effective.

Fungal arthritis is usually indolent but cases of acute arthritis have been reported. ${ }^{19}$ Infection may result from systemic fungal infection or from penetrating cutaneous inoculation by plant material, for example rose thorns. Treatment will often involve drainage and surgical debridement with systemic antifungal agents such as amphotericin B or ketoconazole, although this will depend on the infecting organism and clinical presentation.

Specialist advice should be sought if septic arthritis involves unusual organisms, mycobacteria, or fungi.

Synovial fluid microscopy and culture is an invaluable tool in establishing the diagnosis in patients with septic arthritis. A negative Gram stain and culture result can be misleading even in patients who have not received concomitant antibiotics. In these patients a synovial fluid cell count of $>60000$ cells $/ \mathrm{mm}^{3}$ with a predominance of polymorphs and $>95 \%$ ragocytes will confirm septic arthritis. ${ }^{20}$ Synovial fluid cytology results can be available within 60 minutes if the appropriate laboratory expertise is on site.

\section{CRYSTAL INDUCED ARTHRITIS}

Acute monoarthritis is frequently associated with a number of crystalline and other particulate materials. The clinical appearance of the joint and the presence of fever may make differentiation from septic arthritis virtually impossible. This emphasises the importance of arthrocentesis and careful synovial fluid analysis. Unfortunately the presence of crystals is not pathognomonic of crystal arthritis as they may occur in the context of other forms of arthritis and in infected joints.
Table 4 Conditions which predispose to gout

Hypertension
Hyperlipidaemia
Excess alcohol intake
Renal impairment
Drugs
Increase production of uric acid-for example, cytotoxic
drugs
Reduce uric acid excretion, for example, thiazide and loop
diuretics

Gout

Gout is one of the oldest recorded forms of arthritis and is characterised by recurrent episodes of acute inflammation due to the release of microcrystals of monosodium urate. The incidence increases with age up to a peak in men between 40-50 and women over 60 . The first metatarsophalangeal joint is affected in $90 \%$ of patients (fig 1), although any joint may be involved. The initial episode is monoarticular in $90 \%$ of patients with involvement of the first metatarsophalangeal joint in $50 \%$. Gout is traditionally associated with a high serum urate concentration. A normal concentration does not exclude the diagnosis. ${ }^{21}$ Other predisposing factors are listed in table 4 .

Arthrocentesis should be considered in even the most painful looking joint. The presence of needle shaped crystals which show strong negative birefringence under polarised light microscopy is diagnostic of monosodium urate.

An acute attack of gout is best treated with colchicine $(500 \mu \mathrm{g}$ every six hours or until diarrhoea develops) or a non-steroidal antiinflammatory drug (NSAID). NSAIDs are associated with a significant risk of gastroduodenal ulceration and therefore the choice of drug, dose, and duration should aim at effective treatment with the minimum risk to the patient-that is, safest drug at lowest dose required for shortest possible time. Although a number of NSAIDs are marketed on the strength of their side effect profiles, none is ideal. In patients at a significant risk of side effects, for example the elderly, patients with previous episodes of ulceration, or patients with concomitant cardiac disease (particularly patients on aspirin or warfarin) it is advisable to avoid NSAIDs. If this is not possible then the co-presciption of omeprazole as a gastroprotective agent should reduce their risk. Intra-articular or oral steroids are occasionally needed, although they should be used with caution because of the difficulty in excluding septic arthritis. Potential risk factors should be identified and if possible treated. Ideally antihyperuricaemic drugs (allopurinol) should not be started until an attack of gout has settled, although this may prove impossible in patients who experience frequent attacks.

\section{Calcium pyrophosphate arthritis (pseudogout)}

Crystals of calcium pyrophosphate dihydrate (CPPD) cause an acute arthritis that is clinically indistinguishable from gout, hence the term pseudogout. The incidence of CPPD arthritis increases with age with a female preponderance and a peak in the 60s and 70s. CPPD is the commonest cause of acute 
Table 5 Conditions associated with calcium pyrophosphate arthritis

\begin{tabular}{ll}
\hline Predisposing conditions & Triggering factors \\
\hline Haemochromatosis & Trauma \\
Hyperparathyroidism & Intercurrent illnes \\
Hypomagnesaemia & Joint aspiration \\
Hypophosphatasia & Blood/fluid infusion \\
Wilson's disease & \\
Gout & \\
Ochronosis & \\
\hline
\end{tabular}

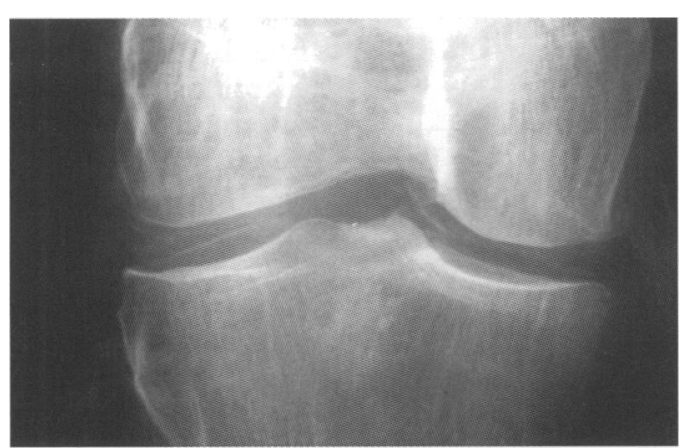

Figure 2 Chondrocalcinosis seen in the knee of a patient with pseudogout.

monoarthritis of the elderly and is often the first manifestation of a previously asymptomatic pyrophosphate arthropathy. The knee is most commonly affected, although any joint may be involved. Concurrent attacks in more than one joint are uncommon and polyarticular attacks rare. Unusual presentations in younger patients suggest a predisposing condition. A number of metabolic conditions which are associated with CPPD arthritis are listed in table 5 along with situations which may trigger an acute attack. Aspirated synovial fluid will often be turbid and blood stained with the appearance of pus. Fever is common, so differentiation from septic arthritis is especially important. A lower ragocyte count may help to differentiate crystal arthritis from septic arthritis. Careful synovial fluid examination should reveal the typical rhomboid or rod-like crystals with weak positive birefringence on polarised light microscopy.

Plain radiographs can be helpful if the typical chondrocalcinosis of fibrocartilage and hyaline cartilage is seen (fig 2 ). The radiological changes associated with pyrophosphate arthropathy are almost identical to those of osteoarthritis with which it often coexists. The joint distribution and presence of exuberant osteophytes and cyst formation may permit distinction.

The aims of treatment are to reduce symptoms and identify and treat triggering illness. Joint aspiration greatly reduces symptoms, although fluid reaccumulation often necessitates a second aspiration followed by steroid injection. Relapsing inflammation can be helped by joint lavage. NSAIDs may provide additional benefit.

Monosodium urate and CPPD crystals are both found in synovial tendon sheaths and bursae and are therefore capable of causing tendinitis or bursitis, which may be indistinguishable from an acute arthritis.
Hydroxyapatite crystal arthritis (calcific periarthritis)

Calcific deposits around the shoulder and other joints are often asymptomatic. Mild trauma or intercurrent illness may precipitate an acute attack of periarthritis, which usually involves the shoulder and may be intensely painful. The condition appears to be initiated by rupture of the calcific deposit leading to crystals being shed and inducing an inflammatory response. Diseases associated with hydroxyapatite deposition include scleroderma, hyperparathyroidism, dermatomyositis, inflammatory joint disease, and osteoarthritis. Patients receiving haemodialysis and chronic ambulatory peritoneal dialysis are also liable to hydroxyapatite deposition.

Plain radiographs will usually demonstrate periarticular calcification. The deposits may however change and disappear during an acute attack only to reappear again later. Aspiration may reveal toothpaste-like material containing calcium deposits and inflammatory material. Individual crystals are too small to be resolved under light microscopy, although clumps may be recognised after staining with alizarin red S.

Symptomatic treatment with NSAIDs is beneficial. Local steroid injections should be avoided as they increase the likelihood of further attacks.

\section{Miscellaneous crystal arthritis}

Oxalate crystals can induce a monoarthritis, although their presence has only been reported in patients with renal failure. ${ }^{22}$

Lipid crystals may induce inflammation in a joint after trauma or haemarthrosis. They are readily identifiable on polarised light microscopy. Cholesterol crystals are seen most commonly as a complication of effusions due to rheumatoid or osteoarthritis.

\section{TRAUMA}

Trauma to a joint leading to internal derangement, haemarthrosis, or even fracture can be mistaken for an acute arthritis. Foreign bodies can cause an acute arthritis. A history of penetrating injury should be sought. Plant thorns, ${ }^{23}$ wood splinters, ${ }^{24}$ sea urchin spines, ${ }^{25}$ and fibre glass have all been reported. Haemarthrosis in the absence of significant trauma should always be investigated further with clotting studies. Osteoarthritis may present with worsening pain and swelling as a result of minor trauma or spontaneous osteonecrosis.

\section{REACTIVE ARTHRITIS}

A wide range of infections are capable of precipitating a reactive arthritis (table 6). A careful history and examination is therefore required to establish both the possible infectious agent and the extent of disease.

Investigations must include a thorough search for the precipitating infection with appropriate serological tests, swabs, and culture. A referral to the department of genitourinary medicine for the exclusion of secondary infections and contact tracing is mandatory if any doubt exists. Synovial fluid aspiration has a diagnostic and therapeutic role. The presence 
Table 6 Infectious causes of reactive arthritis

\begin{tabular}{ll}
\hline Bacteria & Salmonella \\
& Shigella \\
& Yersinia \\
& Campylobacter \\
& Borrelia \\
& Chlamydia \\
& Streptococcal \\
& Rubella \\
Viruses & Hepatitis B \\
& Parvovirus \\
& Epstein-Barr \\
& Cytomegalovirus \\
& HTLV-1 \\
& HIV \\
& Paramyxovirus (mumps) \\
\hline
\end{tabular}

of cytophagocytic macrophages is consistent with reactive arthritis.

The acute monoarthritis should be treated with NSAIDs and intra-articular steroids. Antibiotic treatment should be given where appropriate. A chronic arthritis may develop, ${ }^{26}$ particularly in those patients who are HLA B27 positive. ${ }^{27}$ Consequently an early rheumatology referral is recommended.

The term Reiter's syndrome refers to the classical triad of reactive arthritis, urethritis, and uveitis precipitated by enteric and urogenital infections. Young adults are most commonly affected and there is a clear association with the antigen HLA B27. ${ }^{28}$

Lyme disease is a systemic inflammatory disorder caused by a spirochete, Borrelia burgdorferi, which is transmitted from animal to man by ticks of the Ixodes ricinus complex. Arthritis occurs in $60 \%$ of cases and may present as a monoarthritis, often involving the knee. ${ }^{29}$ The diagnosis should be considered in patients who have visited woodland areas with a deer population. The arthritis will usually occur six months after the primary infection, although it can be the presenting feature. A history of a tick bite followed by the appearance of the pathognomonic rash, erythema chronicum migrans, is diagnostic. A serological response will confirm the diagnosis. Suspected cases should be referred for specialist advice as there are potential long term cardiac, rheumatological, and neurological sequelae. For patients with acute arthritis without cardiac or neurological involvement a 10 day course of amoxycillin (500 mg four times a day) or azithromycin ( $500 \mathrm{mg}$ for first day then $250 \mathrm{mg}$ four times a day for four further days) is usually effective. Patients with systemic manifestations should be referred for specialist advice on treatment.

\section{MALIGNANCY}

An acute monoarthritis may be the first indication of malignancy. Para-articular involvement by malignant tumours arising from bone, such as osteogenic sarcoma, chondrosarcoma or fibrosarcoma, may give rise to a monoarthritis. Plain radiographs, radionuclide bone scans, and magnetic resonance imaging provide helpful diagnostic information. Occasionally a tumour will invade the synovium, malignant cells may then be found in synovial fluid.

Benign tumours such as osteoid osteoma, haemangioma, pigmented villonodular synovi- tis (PVNS), or lipoma can give rise to arthritis. Recurrent attacks of acute pain and swelling of a joint with repeated aspiration of bloody fluid suggests either a haemangioma or PVNS.

An acute monoarthritis in a child always raises the possibility of leukaemia. Synovial fluid cytology may confirm the diagnosis.

Direct invasion of the synovial membrane and the bone by non-haematological malignancy may lead to monoarthritis. The knee is most commonly affected and tumours that have been implicated include breast, lung, gastrointestinal, and malignant melanoma. ${ }^{30}$

A number of paraneoplastic syndromes can present with arthritis. The most notable is hypertrophic osteoarthropathy, which is usually associated with intrathoracic malignancy. Plain radiographs will demonstrate periostitis with subperiosteal new bone formation diagnostic of this condition. The site of tenderness should be taken into account as periostitis may be sufficiently proximal to the joint line as to be overlooked if the radiograph does not include enough of the shaft.

\section{SYSTEMIC DISEASE}

Many systemic diseases may present with an acute monoarthritis. These include inflammatory bowel disease, vasculitic conditions, connective tissue disease, sarcoidosis and chronic inflammatory arthritis, for example rheumatoid arthritis.

\section{Conclusion}

The patient presenting to the $A \& E$ department with an acute monoarthritis provides the clinician with a fascinating diagnostic challenge. The differential diagnosis will include a variety of infections, crystals, multisystem diseases, and malignancies. A thorough history and examination will be required to elucidate the various symptoms and signs. Septic arthritis should be excluded above all other diagnoses. Referral to a rheumatologist, orthopaedic surgeon, or specialist in infectious diseases or genitourinary medicine should be considered. Careful assessment of the patient with monoarthritis and a sound understanding of the potential differential diagnosis will enable the clinician to formulate a working diagnosis in most patients.

We are grateful for the advice and contributions of $\mathrm{Mr} \mathrm{J}$ Wardrope, Consultant in Accident and Emergency Medicine, in the preparation of this manuscript.

Conflict of interest: none.

Funding: none.

\section{Multiple choice questions relating to this} article

(1) What is the commonest cause of bacterial septic arthritis?

- Staphylococcus epidermis

- Neisseria gonorrhoeae

- Haemophilus influenzae

- Staphylococcus aureus

- Streptococcus pneumonia

(2) Which of the following statements about gout is/are true?

- Serum uric acid concentrations are always raised 
- Systemic oral steroids can be effective in polyarticular gout

- Uric crystals demonstrate positive birefringence under polarised light

- Synovial fluid from septic arthritis and gout can be readily distinguished

- The presence of uric acid crystals within synovial fluid confirms gout

(3) Which of the following infections are commonly associated with reactive arthritis?

- Group A streptococci

- Staphylococcus aureus

- Neisseria gonorrhoeae

- Parvovirus B19

- Borrelia burgdorferi

\section{Key references}

The three key references are: Baker and Schumacher, ${ }^{9}$ Freemont and Denton, ${ }^{20}$ and Boss and Seegmiller. ${ }^{21}$ Three further useful references are: Freemont and Denton, ${ }^{5}$ the Report of a joint working group of the British Society for Rheumatology and the Research Unit of the Royal College of Physicians, ${ }^{7}$ and Walker et al. ${ }^{8}$

1 Dubost JJ, Fis I, Denis P, et al. Polyarticular septic arthritis. Medicine 1993;72:296-310.

2 Knight DJ, Gilbert FJ, Hutchison JD. Septic arthritis in osteoarthritic hips. BMF 1996;313:40-1.

3 Doube A, Calin A. Bacterial endocarditis presenting as acute monoarthritis. Ann Rheum Dis 1988;47:598-9.

4 Liang MH, Sturrock RD. Technique of joint aspiration. In: Klippel JH, Dieppe PA, ed. Rheumatology. London: Mosby Klippel JH, Dieppe PA, ed. Rheumatology. L

5 Freemont AJ, Denton J. The role of synovial fluid microscopy in the diagnosis of joint disease. In: Gresham $\mathrm{GA}$, ed. Atlas of synovial fluid cytology. London: Kluwer Academic, 1991: 125-30.

6 Klein MA, Winalski CS, Wax MR, et al. MR imaging of septic sacroiliitis. ₹ Comput Assist Tomogr 1991;15:126-32.

7 Report of a joint working group of the British Society for Rheumatology and the Research Unit of the Royal College of Physicians. Guidelines and a proposed audit protocol for the initial managment of an acute hot joint. $\mathcal{F} R$ Coll Physicians Lond 1992;26:83-5.

8 Walker DJ, Young I, Hassey GA, et al. The acute hot joint in medical practice. $\mathcal{F} R$ Coll Physicians Lond 1995;29:101-4.

9 Baker DG, Schumacher HR. Acute monoarthritis. $N$ Engl f Med 1993;329:1013-19.
10 O'Brien JP, Goldenberg DL, Rice PA. Disseminated gonococcal infection; a prospective analysis of 49 patients and a review of pathophysiology and immunological mechanisms. Medicine 1983;62:395-406.

11 Britigan BE, Cohen MS, Sparling PF. Gonococcal infection: a model of molecular pathogenesis. $N$ Engl $f \mathrm{Med}$ 1985;312:1683-94.

12 Muralidhar B, Rumore PM, Steinman CR. Use of the polymerase chain reaction to study arthritis due to neissepolymerase chain reaction to study arthritis due
ria gonorrhoea. Arthritis Rheum $1994 ; 37: 710-17$.

13 Liebling MR, Arkfield DG, Michelini GA, et al. Identification of neisseria gonorrhoea in synovial fluid using the polymerase chain reaction. Arthritis Rheum 1994;37:702-9.

14 Goldenberg DL, Cohen AS. Acute infectious arthritis: a review of patients with nongonococcal joint infections (with emphasis on therapy and prognosis). Am $\mathcal{F}$ Med 1976;60:369-77.

15 Hall BB, Rosenblatt JE, Fitzgerald RH Jr. Anaerobic septic arthritis and osteomyelitis. Orthop Clin North Am 1984;15: 505-16.

16 Goldenberg DL. Bacterial arthritis. Curr Opin Rheumatol 1994;6:394-400.

17 Boulware DW, Lopez M, Gum OB. Tuberculous podagra. $\mathcal{F}$ Rheumatol 1985;12:1022-4.

18 Bern S, Goldstein M, Bishko F. Clinical and diagnostic features of tuberculous arthritis. Am f Med 1972;53:36-42.

19 Katzenstein D. Isolated candida arthritis: report of a case and definition of a distinct clinical syndrome. Arthritis Rheum 1985;28:1421-4.

20 Freemont AJ, Denton J. Infective arthritis. In: Gresham GA, ed. Atlas of synovial fluid cytology. London: Kluwer Academic, 1991: 106-11.

21 Boss GR, Seegmiller JE. Hyperuricaemia and gout: classification, complications and management. $N$ Engl $f \mathrm{Med}$ 1979;300:1459-68.

22 Reginato AJ, Kurnik BRC. Calcium oxalate and other crystals associated with kidney diseases and arthritis. Semin Arthritis Rheum 1989;18:198-224.

23 Goupille P, Fouquet B, Favard L, et al. Two cases of plant thorn synovitis. Difficulties in diagnosis and treatment. $\mathcal{F}$ thorn synovitis. Difficulties

24 O'Connor CR, Reginato AJ, DeLong WG Jr. Foreign body reactions simulating acute septic arthritis. $\mathcal{f}$ Rheumatol 1988;15:1568-71.

25 Wilson GE, Curry A, Kennaugh JH, et al. Severe granulomatous arthritis due to spinous injury by a sea mouse annelid worm. 7 Clin Pathol 1990;43:291-4.

26 Csonka GW. The course of Reiter's syndrome. BMF 1958;i: 1088-90.

27 Leirisalo-Repo M, Skylv G, Kousa M. Follow-up study of Reiter's disease and reactive arthritis. Factors influencing the natural course and the prognosis. Clin Rheumatol 1987; the natural

28 Colin A, Fries JF. An “experimental” epidemic of Reiter's syndrome revisited. Follow-up evidence on genetic and environmental factors. Ann Intern Med 1976;84:564-6.

29 Steere AC. Musculoskeletal manifestations of Lyme disease. Am $\mathcal{F}$ Med 1995;98:44S-48S

30 Caldwell DS, McCallum RM. Rheumatological manifestations of cancer. Med Clin North Am 1986;70:3 